Gynäkologe 2021 · 54:428-434

https://doi.org/10.1007/s00129-021-04804-6

Angenommen: 9. April 2021

Online publiziert: 4. Mai 2021

(c) Springer Medizin Verlag $\mathrm{GmbH}$, ein Teil von

Springer Nature 2021

\section{Redaktion}

R. Felberbaum, Kempten

M. Sauter, Kempten

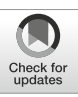

\author{
Arne Simon ${ }^{1} \cdot$ Michael Zemlin $^{2}$ Martina Geipel ${ }^{2}$ Barbara Gärtner ${ }^{3}$. \\ Jakob Armann ${ }^{4}$. Sascha Meyer ${ }^{2}$ \\ 'Pädiatrische Onkologie und Hämatologie, Universitätsklinikum des Saarlandes, Homburg/Saar, \\ Deutschland \\ ${ }^{2}$ Allgemeine Pädiatrie und Neonatologie, Universitätsklinikum des Saarlandes, Homburg/Saar, \\ Deutschland \\ ${ }^{3}$ Institut für Medizinische Mikrobiologie und Hygiene (IMMH), Universität und Universitätsklinikums des \\ Saarlandes, Homburg/Saar, Deutschland \\ ${ }^{4}$ Klinik und Poliklinik für Kinder- und Jugendmedizin, Universitätsklinikum Carl Gustav Carus, Technische \\ Universität Dresden, Dresden, Deutschland
}

\title{
Hygienestandards auf einer neonatologischen Intensivstation
}

kommen häufig Bakterien aus der Krankenhausumgebung hinzu.

\section{Leitlinien und interne SOPs}

Neugeborene, die auf einer neonatologischen Intensivstation (NICU) behandelt werden, sind besonders gefährdet für nosokomiale Infektionen [1-3]. Die Gründe hierfür sind multifaktoriell. Aufseiten der Früh- und Neugeborenen besteht eine Unreife verschiedener Komponenten der Infektionsabwehr. Die Epidermis- und Schleimhautbarriere sehr unreifer Frühgeborener (Geburtsgewicht $<1500$ g, ,very low birth weight", VLBW) in den ersten Lebenswochen ist durchlässiger für Krankheitserreger. Unspezifische Barrieren (z.B. der intakten Haut oder der Atemwege) werden durch invasive Maßnahmen der Intensivtherapie (Katheter, Drainagen, Intubation) durchbrochen. Zudem wird das intensivmedizinisch behandelte Kind im Verlauf mannigfacher Kontakte (Hände!) mit Erregern aus der Umgebung der NICU besiedelt, die bei reifen, zu Hause betreuten Neugeborenen nicht vorkommen. Hinzu kommt der Selektionsdruck durch breit wirksame Antibiotika, durch den sich das Mikrobiom des Kindes verändert. Durch die nicht zu verhindernde Besiedlung des Kindes mit potentiellen Infektionserregern aus dem Krankenhausumfeld besteht gleichzeitig ein erhöhtes Risiko für eine Besiedlung mit multiresistenten Bakterien. In diesem Umfeld bildet sich das Mikrobiom nicht vorrangig aus Bakterien, die ihren Ursprung in der Besiedlung der Mutter haben, sondern es
Die Empfehlungen der Kommission für Krankenhaushygiene und Infektionsprävention (KRINKO [Kommission für Krankenhaushygiene und Infektionsprävention]) beim Robert-Koch-Institut [4-7] stellen einen im $₫ 23$ des Infektionsschutzgesetzes (IfSG) mandatierten Handlungsrahmen bereit, innerhalb dessen die Krankenhaushygiene und Infektionsprävention vor Ort organisiert werden soll. Sein übergeordnetes Vorhandensein garantiert jedoch keineswegs die nachhaltige Implementierung in der klinischen Praxis [8]. Die vor Ort vorhandenen klinikinternen SOPs („standard operating procedures") sollen an aktuelle KRINKO-Empfehlungen angepasst werden. $\mathrm{Zu}$ einzelnen speziellen Themen gibt es zudem ergänzende Leitlinien der Deutschen Gesellschaft für Pädiatrische Infektiologie (DGPI; [9, 10]).

\section{》) Die aktive Beteiligung an der Erstellung interner SOPs erhöht die Wahrscheinlichkeit der Umsetzung}

Wenn alle Entscheidungsträger und das Hygienefachpersonal von Beginn an in die Erstellung von internen SOPs aktiv einbezogen werden, erhöht dies die Wahrscheinlichkeit der späteren Umsetzung (IKEA-Effekt; [11]). Die Einführung von neuen oder überarbeiteten internen SOPs sollte von fallbezogenen und möglichst interaktiven Fortbildungsangeboten begleitet werden, an deren Planung und Durchführung sich das Hygienefachpersonal beteiligen sollte.

Einzelne evidenzbasierte (oder zumindest plausibel belegte) Maßnahmen der Infektionsprävention werden in Präventionsbündeln zusammengefasst, um die Implementierung und nachhaltige Umsetzung zu erleichtern [12-15]. Im Folgenden wird auf Besonderheiten in dieser besonders vulnerablen Patientenpopulation eingegangen.

\section{Prävention gefäßkatheter- assoziierter Infektionen (CLABSI)}

Eine Auswahl von Maßnahmen, die zur Prävention von CLABSI beitragen, fasst - Tab. 1 zusammen $[4,12,13,15]$.

\section{Prävention beatmungs- assoziierter Pneumonien (VAP)}

Eine Auswahl von Maßnahmen zusammen, die zur Prävention von VAP bei Früh- und Neugeborenen beitragen, fasst - Tab. 2 zusammen [14-21]. 
Tab. 1 Komponenten eines CLABSI(gefäßkatheterassoziierte Infektionen)-Präventionsbündels in der Neonatologie [4]

Indikation und Notwendigkeit der weiteren Verwendung zentralvenöser Zugänge tgl. überprüfen, Liegedauer möglichst kurz halten

Maximale Barrierevorkehrungen bei der Anlage eine zentralvenösen Katheters (meist perkutaner Einschwemmkatheter oder Nabelvenenkatheter)

Händedesinfektion vor jeder Manipulation

Gezielte Hautantisepsis ${ }^{\mathrm{a}}$ bei Anlage und bei jedem Verbandswechsel

Vieraugenprinzip bei Anlage, Verbandswechsel, Systemwechsel

Steriler transparenter Folienverband, Routinewechsel erst nach mind. 7 Tagen

Antiseptische Behandlung von Hubs und Zuspritzstellen vor jeder Manipulation mit Alkoholtüchern (z. B. Isopropanol 70\%/Chlorhexidin 2\%; keine Sprühdesinfektion im Inkubator!)

Systemwechsel max. alle $96 \mathrm{~h}$ (ggf. 0,2- $\mu \mathrm{m}$-Partikel- und Bakterienfilter mit $96 \mathrm{~h}$ Standzeit und Endotoxinrückhalt) ${ }^{\mathrm{b}}$

Streng aseptisches Arbeiten bei der Rekonstitution und Zubereitung von Parenteralia, Herstellung komplexer Mischinfusionen im Reinraum (Apotheke)



wirkzeit mindestens 1 min; danach Octenidin 0,1\%/Isopropanol $70 \%$

benn komplexe Mischinfusionen nicht im Reinraum der Apotheke hergestellt werden

\section{Kolonisationsscreening}

Das von der KRINKO empfohlene wöchentliche Kolonisationsscreening [1, 5, 6, 22] wird nach den Ergebnissen eines aktuellen Surveys [8] von den meisten NICUs konsequent umgesetzt. Die hieraus gewonnen Befunde zur Besiedlung mit MRE (multiresistente Erreger) werden bei der Auswahl der empirisch eingesetzten Antibiotikatherapie der Late-onset-Sepsis genutzt [23], was keinesfalls zwingend zu einem häufigeren Einsatz von Reserveantibiotika führt [24]. Dieses Instrument dient auch zur frühzeitigen Erkennung von nosokomialen Übertragungen. Die Besiedlung mehrerer Patienten mit dem gleichen Erreger darf jedoch nicht mit einem Ausbruch verwechselt werden, für den mindestens 2 Infektionen in einem epidemiologischen Zusammenhang Voraussetzung sind [25].

\section{》) Probleme bestehen nach wie vor durch Personalmangel und/oder baulich-funktionelle Limitationen}

Erhebliche Probleme ergeben sich in vielen NICUs weiterhin aufgrund von Personalmangel und/oder baulich-funktionellen Limitationen. Mitunter kann daher keine angemessene Isolierung oder Kohortierung der Patienten erfolgen, die mit bestimmten MRE besiedelt oder infiziert sind [8, 26]. Zurzeit wird eine prospektive, Cluster-randomisierte, multizentrische Studie zur Klärung der Frage durchgeführt, ob bei der Kontaktisolierung von Neugeborenen, die mit 2MRGN NeoPäd (multiresistente gramnegative Erreger, die eine Betalaktamase mit erweitertem Wirkspektrum bilden) besiedelt sind, Standardhygienemaßnahmen zur Vermeidung nosokomialer Übertragungen ausreichen (Studienleiter Prof. Dr. Christoph Härtel, Würzburg).

Gegenstand intensiver Diskussionen (auch zwischen Geburtshelfern und Neonatologen) ist die Empfehlung der KRINKO zur mikrobiologischen Untersuchung von Schwangeren mit drohender Frühgeburt [5]. Entsprechende Besiedlungsdaten der Mutter können sich - beispielsweise bei einer Besiedlung mit MRGN - auf die empirische Therapie des Neugeborenen bei Verdacht auf eine Early-onset-Sepsis auswirken [27, 28]. Ist dieSchwangere MRSA(Methicillin-resistenter Staphylococcus aureus)-besiedelt ${ }^{1}$, stellt sich zum einen die Frage nach der bestmöglichen perioperativen Antibiotikaprophylaxe bei Sectio. Des Weiteren kann sich - bei fehlender Kenntnis -

\footnotetext{
1 Auch in Kliniken der Gynäkologie und Geburtshilfe sollte es definierte Indikationen für ein MRSA-Aufnahmescreening geben.
}

Tab. 2 Komponenten eines

VAP(beatmungsassoziierte Pneumonie)-

Präventionsbündels in der Neonatologie

Einsatz nichtinvasiver Methoden der Atemunterstützung und tgl. Überprüfung der Beatmungsindikation, frühe Extubation anstreben

Akzidentelle Tubusdislokationen mit der Notwendigkeit der Reintubation vermeiden Händedesinfektion plus saubere Einmalhandschuhe vor jeder Manipulation am Beatmungssystem

Wenn möglich: leichte Oberkörperhochlagerung $\left(30^{\circ}\right)$

Kondenswasser im Beatmungssystem vor jeder Umlagerung des Patienten ablassen Wechsel der Beatmungsschläuche im Regelfall alle 7 Tage (solange funktionstüchtig und nicht sichtbar verunreinigt)

Geschlossene Absaugsysteme oder sterile Absaugkatheter (sterile Handschuhe, sterile Kochsalzlösung) beim offenen Absaugen

Mundpflege alle 2-4h (keine Antiseptika, sondern steril filtriertes oder abgekochtes Wasser; kein Tee, kein stilles Mineralwasser)

nicht nur eine MRSA-Übertragung auf das Neugeborene, sondern auch eine nosokomiale Ausbreitung innerhalb der NICU ereignen [29].

\section{Muttermilch und Formula- nahrung}

Muttermilch kann bakterielle und virale Erreger enthalten, die auf das Neugeborene und (bei ungeschütztem Kontakt) auch auf das Personal übertragen werden können. Das gilt auch für die Verabreichung von Muttermilch (oder Formulanahrung) über eine Magensonde mit Hilfe von Einmalspritzen, weshalb hier zusätzlich zur hygienischen Händedesinfektion das Tragen von sauberen Einmalhandschuhen empfohlen wird [30]. Insofern benötigen Kinderkliniken nicht nur für Formulanahrung und Sondenkost, sondern auch für den Umgang mit abgepumpter Muttermilch einen Hygienestandard.

Wasserbäder („Flaschenwärmer“) zum Aufwärmen der Nahrung sind obsolet. In Pulverform vom Hersteller gelieferte Formulanahrung für Neugeborene ist nicht steril, sondern kann bei Einhaltung der Richt- und Warnwerte mit Bakterien kontaminiert sein. Besonders 
kritisch ist in diesem Zusammenhang die Kontamination von Formulanahrung mit dem Erreger Cronobacter (vormals Enterobacter) sakazakii, der durch seine Temperaturtoleranz den Herstellungsprozess überlebt und bei Neugeborenen eine Sepsis mit Meningitis auslösen kann. Daher wird für VLBW-Frühgeborene die Verwendung steriler Formulanahrung empfohlen.

\section{Ausbruchserkennung und -management}

Infektionsausbrüche in der NICU unterscheiden sich mitunter ganz wesentlich von entsprechenden Ereignissen in der Erwachsenenmedizin [29, 31-33]. Darauf muss das lokale Ausbruchsmanagementteam vorbereitet sein.

\section{Clostridioides difficile}

Die pädiatriespezifischen Besonderheiten der C.-difficile-assoziierten Infektionen im Kindesalter [34] wurden in der entsprechenden KRINKO-Empfehlung berücksichtigt [35].

Aus krankenhaushygienischer Sicht ist es wichtig $\mathrm{zu}$ beachten, dass ein hoher Anteil von intensivmedizinisch behandelten Früh- und Neugeborenen toxinbildende $C$. difficile ausscheidet, ohne dass es (bis etwa zum Alter von 13 Monaten) zu einer hiermit assoziierten Erkrankung kommt [36]. Obwohl eine Übertragung auf ältere Patienten der NICU bzw. gemischten pädiatrischen Intensivstation prinzipiell möglich ist, wurde bisher nicht über entsprechende Ausbrüche berichtet [37].

\section{SARS-CoV-2}

Im Register für stationär behandelte Kinder und Jugendliche mit SARS-CoV$2($, severe acute respiratory syndrome coronavirus 2“)-Infektion der DGPI (koordiniert von Dr. Jakob Armann und Prof. Dr. Reinhard Berner, Dresden) sind bis zum 24. Januar 2021 von insgesamt 902 vollständig dokumentierten Verläufen bei Kindern $9 \%$ Neugeborene ( $n=82$; entspricht $23 \%$ der unter $1 \mathrm{Jahr}$ alten Kinder). Zehn (13\%) sind Frühgeborene, das Geschlechterverhältnis ist ausgeglichen. Lediglich 17 (20\%) der Neugeborenen waren während des gesamten stationären Aufenthaltes asymptomatisch, während die übrigen vor allem Fieber und/oder obere Atemwegssymptome zeigten. Allerdings konnte auch bei immerhin 8 Kindern zusätzlich eine bakterielle Infektion (Blutstrominfektion/Pyelonephritis) nachgewiesen werden. Die große Mehrheit (83\%) konnte bereits ohne Folgeschäden wieder entlassen werden. $\mathrm{Zu}$ diesem Thema sind interdisziplinäre Konsensusempfehlungen der Fachgesellschaften erarbeitet worden [38, 39]. Intrauterin/intrapartal exponierte Neugeborene auf der NICU sollten mit den entsprechenden Schutzmaßnahmen versorgt werden, um eine Transmission innerhalb der NICU zu vermeiden. Neugeborene SARS-CoV-2positiver Mütter werden nur dann von ihrer Mutter getrennt, wenn der Gesundheitszustand der Mutter oder des Kindes so kritisch ist, dass ein Roomingin mit zusätzlichen Schutzmaßnahmen nicht realisiert werden kann [38, 39].

Die NICU gehört zu den klinischen Abteilungen, deren Patienten und Mitarbeiter besonders konsequent vor einem vermeidbaren Eintrag von SARS-CoV-2 geschützt werden müssen (hoch vulnerable Bereiche). Das Patientenaufkommen von Level-1-NICUs ist zum größten Teil nicht elektiv steuerbar, und die Kontinuität der intensivmedizinischen Behandlung Früh- und Neugeborener muss auch während der Pandemie gewährleistet bleiben. SARS-CoV-2-Infektionen beim Behandlungsteam könnten diese kritische Infrastruktur [40, 41] sehr rasch in Bedrängnis bringen. Dies gilt auch für SARS-CoV-2-Nachweise bei Patienten oder Eltern, weil diese ein komplexes Kontakt-Tracing und ggf. Quarantäneverordnungen nach sich ziehen [42].

Im Universitätsklinikum des Saarlandes wird der Zugang zur NICU nur noch einem definierten Kreis von absolut notwendigen Mitarbeitern erlaubt. Das Personal wird mit einem PCR(Polymerasekettenreaktion)-basierten Pooling-Verfahren wöchentlich auf SARS-CoV-2 getestet [43]. Organisatorisch schwieriger $\mathrm{zu}$ realisieren ist die SARS-CoV-2-Testung bei den Eltern der Kinder, die nicht als Besucher gel-
Gynäkologe 2021 · 54:428-434

https://doi.org/10.1007/s00129-021-04804-6

( $)$ Springer Medizin Verlag GmbH, ein Teil

von Springer Nature 2021

A. Simon · M. Zemlin · M. Geipel ·

B. Gärtner · J. Armann · S. Meyer

\section{Hygienestandards auf einer neonatologischen Intensivstation}

\section{Zusammenfassung}

In diesem Beitrag werden einige Besonderheiten der Infektionsprävention bei intensivmedizinisch behandelten Früh und Neugeborenen dargestellt. Ergänzend finden sich Hinweise zum krankenhaushygienischen Management der SARS-CoV-2(,severe acute respiratory syndrome coronavirus 2")-Pandemie und zur Antibiotic Stewardship in der neonatologischen Intensivmedizin.

\section{Schlüsselwörter}

Frühgeborene $\cdot$ SARS-CoV-2 $\cdot$ Antimicrobial Stewardship - Clostridioides difficile . Gastrointestinales Mikrobiom · Nosokomiale Infektion · Präventionsbündel

\section{Infection prevention in neonatal intensive care units}

\section{Abstract}

This article focuses on particular aspects of infection prevention concerning the treatment of high-risk neonates in a neonatal intensive care unit (NICU). Furthermore, some aspects regarding severe acute respiratory syndrome coronavirus 2 (SARS-CoV-2) prevention and management in this setting are included. In addition we emphasize the important role of antibiotic stewardship in the NICU.

\section{Keywords}

Preterm infants - SARS-CoV-2 - Antimicrobial stewardship - Clostridioides difficile .

Gastrointestinal microbiome - Nosocomial infection - Preventive bundle

ten, sondern deren Anwesenheit auf der NICU zu definierten Zeiten erforderlich ist. Auch diese werden mindestens einmal pro Woche gescreent. Selbstverständlich dürfen symptomatische Eltern die NICU nicht betreten und alle tragen zusätzlich zur hygienischen Händedesinfektion einen medizinischen MundNasen-Schutz. Interessanterweise wirkt 
Hier steht eine Anzeige.

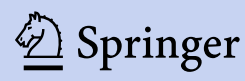


sich die strikte Beachtung der "Corona-Regeln“ im Krankenhaus (und der AHA + L[Abstand, Hygiene, Alltagsmaske + Lüften]-Regeln außerhalb des Krankenhauses!) günstig auf die Inzidenz anderer (nosokomialer) viraler Atemwegsinfektionen bei Frühgeborenen aus. Dies gilt insbesondere für Atemwegsinfektionen durch RS(„,respiratory syncytial“)- und Influenzaviren [44].

\section{Antibiotic Stewardship (ABS) in der Neonatologie}

Die Zunahme multiresistenter Infektionserreger und die unerwünschten Effekte einer breit wirksamen, prolongierten Antibiotikatherapie bei Neugeborenen haben dazu geführt, dass die Indikationsstellung zur Behandlung mit Antibiotika zunehmend kritisch analysiert und ggf. einer infektiologisch fundierten Neubewertung unterzogen wird. Infektionsprävention und ABS sollten sich gegenseitig ergänzen.

\section{》) Infektionsprävention und $A B S$ sollten sich gegenseitig ergänzen}

Der zu breite und unangemessen lange Einsatz von Antibiotika kann negative Konsequenzen für den Patienten selbst [45-49] und für das Erreger- und Resistenzprofil der NICU haben [50-52]. Mikrobiomstudien zeigen, dass der Einsatz von Antibiotika im frühen Kindesalter mit langfristigen unerwünschten Effekten assoziiert ist [53-55]. Selbstverständlich gibt es Neonatologen, die Antibiotika im stationären Setting restriktiv, gezielt und leitlinienkonform einsetzen [56-58]. Insgesamt kann jedoch davon ausgegangen werden, dass mindestens $30 \%$ der Antibiotikaanwendungen in diesem klinischen Setting nicht indiziert sind oder nicht adäquat erfolgen [59-62]. Im klinischen Alltag ist die Verordnung von Antibiotika „Tagesgeschäft". Oft kommen dabei Regime zur Anwendung, die lange tradiert sind („Haben wir schon immer so gemacht") oder für die keine klare Evidenz, sondern lediglich eine abteilungsinterne „Verschreibungsetikette“ besteht („Der
Oberarzt will das so"; [63]). Eine nachhaltige Verbesserung der bisherigen Praxis erfordert eine grundlegende Verhaltensänderung der verantwortlichen Ärzte $[64,65]$. Strukturell-organisatorische Voraussetzungen und konkrete Abläufe des Antibiotikaeinsatzes im Krankenhaus geraten zunehmend in den Fokus einer kritischen Analyse. Hierzu wird auf aktuelle AWMF(Arbeitsgemeinschaft der Wissenschaftlichen Medizinischen Fachgesellschaften)-Leitlinien verwiesen $[66,67]$.

\section{Fazit für die Praxis}

\section{- Krankenhaushygiene und Infektions- prävention in der neonatologischen Intensivmedizin sind ein komplexes Unterfangen mit vielen Besonder- heiten im Vergleich zu anderen Patientenpopulationen. \\ - Die entsprechenden Aufgaben kön- nen nur durch ein gut kooperieren- des, interdisziplinär aufgestelltes Team erfolgreich bewältigt werden. - Das gilt in gleicher Weise für die ABS (Antibiotic Stewardship) in der NICU (neonatologische Intensivstation).}

\section{Korrespondenzadresse}

Prof. Dr. Arne Simon

Pädiatrische Onkologie und Hämatologie, Universitätsklinikum des Saarlandes Kirrberger Str. 9, Geb. 9, 66421 Homburg/Saar, Deutschland

Arne.Simon@uks.eu

\section{Einhaltung ethischer Richtlinien}

Interessenkonflikt. A. Simon ist 2. Vorsitzender der Deutschen Gesellschaft für Pädiatrische Infektiologie. Er leitet gemeinsam mit Prof. Dr. Dr. Soeren Becker das sektorübergreifende Infectio ${ }^{\text {Saar }}$ Netzwerk sowie das pädiatrisch-infektiologische Paedine ${ }^{\text {Saar }}$ Netzwerk. Prof. Simon hat von folgenden Firmen Honorare für Vorträge, Beratertätigkeiten, Reisekosten und über Drittmittelkonten seiner Arbeitgeber abgerechnete Fördermittel für klinische Studien erhalten: AbbVie GmbH Wiesbaden, Sobi, Astra Zeneka, Gilead, Wyeth, Pfizer, Zembon, Beckton Dickenson, 3M Deutschland und MSD. Seit 2004 ist er berufenes Mitglied der KRINKO und koordiniert dort die Arbeitsgruppe neonatologische Intensivmedizin. M.Zemlin hat federführend die AWMF-Leitlinie 0024-008 „Bakterielle Infektionen des Neugeborenen" überarbeitet und war Expertenmitglied bei der Erstellung des IQWIGBerichtes „Screening auf Streptokokken der serologischen Gruppe B im Rahmen der Vorsorgeuntersu- chungen gemäß Mutterschafts-Richtlinien" für den Gemeinsamen Bundesausschuss. Er erhielt Vortragshonorare von Chiesi und Biotest. J. Armann koordiniert die beiden von der DGPI initiierten und inzwischen unter Leitung von Prof. Berner in Dresden fortgeführten Register zu stationär behandelten Kindern und Jugendlichen mit SARSA-CoV-2-Infektion und mit PIMS. Er hat ein Vortraghonorar von Chiesi erhalten. S. Meyer ist Leiter der multizentrischen, von der DFG geförderten Studie NeoVitaA-Studie (ME 3827/1-1/2) und Leiter der "UVC - You Will See Study" (DRKS-ID: DRKS00022262). M. Geipel und B. Gärtner geben an, dass kein Interessenkonflikt besteht.

Für diesen Beitrag wurden von den Autoren keine Studien an Menschen oder Tieren durchgeführt. Für die aufgeführten Studien gelten die jeweils dort angegebenen ethischen Richtlinien.

\section{Literatur}

1. Christoph J, Dame C, Eckmanns T, Gärtner B, Geffers C, Gille C et al. Risikocharakterisierung intensivmedizinisch behandelter Früh- und Neugeborener und Daten zur Ist-Situation in deutschen neonatologischen Intensivpflegestationen 2013 - Fachliche Erläuterungen zu folgender Empfehlung: Praktische Umsetzung sowie krankenhaushygienische und infektionspräventive Konsequenzen des mikrobiellen Kolonisationsscreenings bei intensivmedizinisch behandelten Früh- und Neugeborenen Ergänzende Empfehlung der Kommission für Krankenhaushygiene und Infektionsprävention (KRINKO) beim Robert KochInstitut, Berlin zur Implementierung der Empfehlungen zur Prävention nosokomialer Infektionen bei neonatologischen Intensivpflegepatienten mit einem Geburtsgewicht unter $1.500 \mathrm{~g}$ aus dem Jahr 2007 und 2012 (Epidemiologisches Bulletin 42/2013). Epidemiol Bulletin des Robert KochInstituts, Berlin. 2013;Supplement zu Ausgabe 42(21. Oktober 2013).

2. Härtel C, Gille C, Orlikowsky T (2014) Kolonisation oder Infektion bei Früh- und Neugeborenen - Warum sind sie so gefährdet? Monatsschr Kinderheilkd 162(5):394-402

3. Geffers C, Haller S, Heller G, Gortner L, Göpel W, Bührer C (2014) Nosokomiale Infektionen bei Neugeborenen - Wo stehen wir in Deutschland? Monatsschr Kinderheilkd 162(5):385-393

4. Kommission für Krankenhaushygiene und Infektionsprävention beim Robert Koch Institut Berlin (2018) Prävention von Gefäßkatheter-assoziierten Infektionen bei Früh- und Neugeborenen - Empfehlung der Kommission für Krankenhaushygiene und Infektionsprävention (KRINKO) beim Robert Koch Institut. Bundesgesundheitsblatt Gesundheitsforschung Gesundheitsschutz 61(5):608-626

5. Kommission für Krankenhaushygiene und Infektionsprävention beim Robert Koch-Institut B. Praktische Umsetzung sowie krankenhaushygienische und infektionspräventive Konsequenzen des mikrobiellen Kolonisationsscreenings bei intensivmedizinisch behandelten Früh- und Neugeborenen - Ergänzende Empfehlung der KRINKO beim Robert Koch-Institut, Berlin, zur Implementierung der Empfehlungen zur Prävention nosokomialer Infektionen bei neonatologischen Intensivpflegepatienten mit einem Geburtsgewicht unter $1.500 \mathrm{~g}$ aus dem Jahr 2007 und 2012. Epidemiol Bulletin des Robert Koch-Instituts, Berlin. 2013;Nr. 42(21. Oktober 2013):421-33. 
6. Kommission für Krankenhaushygiene und Infektionsprävention beim Robert Koch-Institut B. Ergänzende Empfehlung (2012) zur „Prävention nosokomialer Infektionen bei neonatologischen Intensivpflegepatienten mit einem Geburtsgewicht unter $1.500 \mathrm{~g}^{\prime \prime}$ (2007). Epidemiol Bulletin des Robert Koch-Instituts, Berlin. 2012(16. Januar 2012 /Nr. 2):13-5.

7. Kommission für Krankenhaushygiene und Infektionsprävention beim Robert Koch-Institut (2007) Empfehlung zur Prävention nosokomialer Infektionen bei neonatologischen Intensivpflegepatienten miteinem Geburtsgewicht unter $1500 \mathrm{~g}$. Bundesgesundheitsblatt Gesundheitsforschung Gesundheitsschutz 50(10):1265-1303

8. Litz JE, Goedicke-Fritz S, Hartel C, Zemlin M, Simon A (2019) Umsetzung des mikrobiologischen Kolonisationsscreenings: Umfrage an 80 neonatologischen Intensivstationen. Epidemiol Bulletin des Robert Koch-Instituts. RKI, Berlin, S 387-392 (12. September 2019)

9. Deutsche Gesellschaft für Pädiatrische Infektiologie (2014) Infektionspräventives Vorgehen bei Nachweis von MRGN im Kindesalter - Empfehlung der Arbeitsgruppe MRGN der Deutschen Gesellschaft für Pädiatrische Infektiologie und des Paed ICProjektes. Hyg Med 39(10):392-399

10. Arbeitsgruppe MRSA der Deutschen Gesellschaft für Pädiatrische Infektiologie und des Paed IC Projektes (2014) Umgang mit MRSA-Nachweisen bei ansonsten gesunden Kindern ohne Infektionszeichen (Neugeborene und ambulantes Betreuungsumfeld). Hyg Med 39(9):344-357

11. Norton M, Ariely D (2012) The IKEA effect: when labor leads to love. J Consum Psychol 22(3):453-560

12. Schmid S, Geffers C, Wagenpfeil G, Simon A (2018) Preventive bundles to reduce catheter-associated bloodstream infections in neonatal intensive care. GMS Hyg Infect Control 13:Doc10. https://doi.org/ 10.3205/dgkh000316

13. Bierlaire S, Danhaive O, Carkeek K, Piersigilli F (2021) How to minimize central line-associated bloodstream infections in a neonatal intensive care unit: a quality improvement intervention based on a retrospective analysis and the adoption of an evidence-based bundle. Eur J Pediatr 180(2):449-460. https://doi.org/10.1007/s00431020-03844-9

14. Goerens A, Lehnick D, Buttcher M, Daetwyler K Fontana M, Genet P et al (2018) Neonatal ventilator associated pneumonia: a quality improvement initiative focusing on antimicrobial stewardship. Front Pediatr 6:262. https://doi.org/10.3389/fped. 2018.00262

15. Smulders CA, van Gestel JP, Bos AP (2013) Are central line bundles and ventilator bundles effective in critically ill neonates and children? Intensive Care Med 39(8):1352-1358. https://doi. org/10.1007/s00134-013-2927-7

16. Simon A, Tutdebi E, Gortner L (2011) Beatmungsassoziierte Pneumonie bei Kindern. Monatsschr Kinderheilkd 159(3):224-232

17. Simon A (2017) Kapitel 5: Beatmungsassoziierte Pneumonie im Kindesalter - Prävention, Diagnostik und Therapie. In: Humberg A, Herting $E_{\text {, }}$ Göpel W, Härtel C (Hrsg) Beatmung in Pädiatrie und Neonatologie Ein Leitfaden für Pädiater, Neonatologen und Anästhesisten, 1. Aufl. Thieme, Stuttgarthttps://doi.org/10.1055/b-004-129744

18. Iosifidis E, Pitsava G, Roilides E (2018) Ventilatorassociated pneumonia in neonates and children: a systematic analysis of diagnostic methods and prevention. Future Microbiol 13:1431-1446. https://doi.org/10.2217/fmb-2018-0108

19. Azab SF, Sherbiny HS, Saleh SH, Elsaeed WF, Elshafiey MM, Siam AG et al (2015) Reducing ventilator-associated pneumonia in neonatal intensive care unit using "VAP prevention Bundle" a cohort study. BMC Infect Dis 15:314. https://doi. org/10.1186/s12879-015-1062-1

20. Ceballos K, Waterman K, Hulett T, Makic MB (2013) Nurse-driven quality improvement interventions to reduce hospital-acquired infection in the NICU. Adv Neonatal Care 13(3):154-163. https://doi.org/ 10.1097/ANC.0b013e318285fe70 (quiz 64-5)

21. Weber CD (2016) Applying adult ventilator-associated pneumonia bundle evidence to the ventilated neonate. Adv Neonatal Care 16(3):178-190. https://doi.org/10.1097/anc.0000000000000276

22. Scheithauer S, Simon A (2015) Mikrobiologisches Kolonisationsscreening bei intensivmedizinisch behandelten Früh- und Neugeborenen. Krankenhhyg up2date 10(4):265-274. https://doi.org/10. 1005/s-005-30746

23. Litz JE, Goedicke-Fritz $S$, Hartel C, Zemlin M, Simon A (2019) Management of early- and lateonset sepsis: results from a survey in $80 \mathrm{German}$ NICUs. Infection 47:557-564. https://doi.org/10. 1007/s15010-018-1263-9

24. Schoendorf D, Simon A, Wagenpfeil G, Gärtner B, Geipel M, Zemlin M et al (2020) Colonization screening targeting multidrug-resistant gramnegative pathogens does not increase the use of carbapenems in very low birth weight infants. Front Pediatr 8:427. https://doi.org/10.3389/fped. 2020.00427. eCollection 2020

25. Schulz-Stubner S, Zimmer P, Leonards P, Knipp U, Michels H, Kunitz O et al (2015) Colonizationoutbreak of two clonally different strains of Serratia marcescens in a neonatal intensive care unit. Bundesgesundheitsblatt Gesundheitsforschung Gesundheitsschutz 58(2):190-196. https://doi. org/10.1007/s00103-014-2099-9

26. Haertel C, Simon A, Geffers C, Schaper A, Herting E, Göpel W et al (2013) Nosokomiale Infektionen bei Frühgeborenen - Umsetzung der KRINKOEmpfehlungen im Deutschen Frühgeborenennetzwerk. Monatsschr Kinderheilkd 161(1):27-33

27. Bulabula ANH, Dramowski A, Mehtar S, Danino D Melamed R, Sterer B et al (2019) Transmission of multidrug-resistant Gram-negative bacteria from colonized mothers to their infants: a systematic review and meta-analysis Mother-to-child transmission of extended-spectrum-beta-lactamaseproducing Enterobacteriaceae. J Hosp Infect 100(1):40-46. https://doi.org/10.1016/j.jhin.2019. 10.001

28. Danino D, Melamed R, Sterer B, Porat N, Hazan G, Gushanski A et al (2018) Mother-to-child transmission of extended-spectrum-beta-lactamaseproducing Enterobacteriaceae. J Hosp Infect 100(1):40-46. https://doi.org/10.1016/j.jhin.2017. 12.024

29. Kraus-Haas M, Mielke M, Simon A (2015) Update on outbreaks reported from neonatal intensive care units (2010-203): staphylococcus aureus. Bundesgesundheitsblatt Gesundheitsforschung Gesundheitsschutz 58(3):323-338. https://doi. org/10.1007/s00103-014-2115-0

30. Berthelot $P$, Grattard F, Patural H, Ros A, JelassiSaoudin H, Pozzetto B et al (2001) Nosocomial colonization of premature babies with Klebsiella oxytoca: probable role of enteral feeding procedure in transmission and control of the outbreak with the use of gloves. Infect Control Hosp Epidemiol 22(3):148-151
Hier steht eine Anzeige. Springer 
31. Gastmeier P (2014) Serratia marcescens: an outbreak experience. Front Microbiol 5:81. https:// doi.org/10.3389/fmicb.2014.00081

32. Gastmeier P, Loui A, Stamm-BalderjahnS, HansenS, Zuschneid I, Sohr D et al (2007) Outbreaks in neonatal intensive care units - they are not like others. Am J Infect Control 35(3):172-176

33. Kraus-Haas M, Mielke M, Simon A (2015) Update on outbreaks reported from neonatal intensive care units: Serratia marcescens, Klebsiella pneumoniae, Acinetobacter baumannii, and Pseudomonas aeruginosa. Bundesgesundheitsblatt Gesundheitsforschung Gesundheitsschutz 58(3):308-322. https://doi.org/10.1007/s00103014-2114-1

34. Weichert S, Simon A, von Müller L, Adam R, Schroten H (2015) Clostridium-difficile-assoziierte Infektionen im Kindes- und Jugendalter. Monatsschr Kinderheilkd 163(5):427-436

35. Kommission für Krankenhaushygiene und Infektionsprävention beim Robert Koch Institut. (2019) Hygienemaßnahmen bei Clostridioides difficileInfektion (CDI) Empfehlung der Kommission für Krankenhaushygiene und Infektionsprävention (KRINKO) beim Robert Koch-Institut. Bundesgesundheitsblatt Gesundheitsforschung Gesundheitsschutz 62(7):906-923. https://doi. org/10.1007/s00103-019-02959-1

36. Enoch DA, Butler MJ, Pai S, Aliyu SH, Karas JA (2012) Clostridium difficile in children: colonisation and disease. J Infect 63(2):105-113. https://doi.org/10. 1016/j.jinf.2011.05.016

37. Kociolek LK, Gerding DN, Espinosa RO, Patel SJ, Shulman ST, Ozer EA (2018) Clostridium difficile whole genome sequencing reveals limited transmission among symptomatic children: a singlecenter analysis. Clin Infect Dis 67(2):229-234. https://doi.org/10.1093/cid/ciy060

38. Deutsche Gesellschaft für Pädiatrische Infektiologie.Stellungnahme der Deutschen Gesellschaft für pädiatrische Infektiologie(DGPI) im Einvernehmen mit der Deutschen Gesellschaft für Gynäkologie und Geburtshilfe (DGGG) und der Deutschen GesellschaftfürPerinatale Medizin (DGPM)-Umgang mit Neugeborenen SARS-CoV-2 positiver Mütter mit oder ohne klinische Erkrankung (COVID19). https://dgpi.de/stellungnahme-dgpi-dgggdgpm-umgang-mit-neugeborenen-sars-cov-2positiver-muetter/.2020;31. März 2020.

39. Deutsche Gesellschaft für Perinatale Medizin (DGPM), Deutsche Gesellschaft für Gynäkologie und Geburtshilfe (DGGG), Deutsche Gesellschaft für Pränatal- und Geburtsmedizin (DGPGM), Deutsche Gesellschaft für Pädiatrische Infektiologie (DGPI), Gesellschaft für Neonatologie und Pädiatrische Intensivmedizin (GNPI), Nationale Stillkommission (NKS). Update 10/2020 Empfehlungen zu SARS-CoV-2/COVID-19 in Schwangerschaft, Geburt und Wochenbett https://idw-online.de/ de/attachmentdata80958.2020.

40. Gemeinsamer Bundesausschuss (GbA). Beschluss des Gemeinsamen Bundesausschusses über eine Änderung der Qualitätssicherungs-RichtlinieFrühund Reifgeborene/QFR-RL:(§7Nachweisverfahren und Anlage 2 Anforderung zum Pflegepersonal) 2016;15. http://www.english.g-ba.de/ (Erstellt: 12.2016).Zugegriffen:26. Apr. 2021

41. Gemeinsamer Bundesausschuss gemäß 91 Abs.7 SGB V. Richtlinie des Gemeinsamen Bundesauschusses über Maßnahmen zur Qualitätssicherung für die stationäre Versorgung von Kindern und Jugendlichen mit hämato-onkologischen Krankheiten gemäß § 137 Abs. 1 Satz 1 Nr. 2 SGB V für nach SGB V zugelassene Krankenhäuser in der Fassung vom 1. Mai 2006 veröffentlicht im Bundesanzeiger 2006 (S. 4 997) in Kraft getreten am 1. Januar 2007 zuletzt geändert am 18. Oktober 2012 veröffentlicht im Bundesanzeiger (BAnz AT 13. Dez. 2012 B3) in Kraft getreten am 1. Januar 2013. 2006;http://www.g-ba.de/informationen/ richtlinien/47/

42. Kabesch $M$, Roth $S$, Brandstetter $S$, Häusler $S$, Juraschko E, Weigl M et al (2020) Successful containment of Covid-19 outbreak in a large maternity and perinatal center while continuing clinical service. Pediatr Allergy Immunol 31(5):560-564. https://doi.org/10.1111/pai.13265

43. Lohse S, Pfuhl T, Berkó-Göttel B, Rissland J, Geißler T, Gärtner B et al (2020) Pooling of samples for testing for SARS-CoV-2 in asymptomatic people. Lancet Infect Dis. https://doi.org/10.1016/ s1473-3099(20)30362-5

44. Tong WY, Yung CF, Chiew LC, Chew SB, Ang LD, Thoon KC et al (2020) Universal face masking reduces respiratory viral infections among inpatient very-low-birthweight neonatal infants. Clin Infect Dis 71(11):2958-2961. https://doi.org/10.1093/ cid/ciaa555

45. Alexander VN, Northrup V, Bizzarro MJ (2011) Antibiotic exposure in the newborn intensive care unit and the risk of necrotizing enterocolitis. JPediatr 159(3):392-397.https://doi.org/10.1016/ j.jpeds.2011.02.035

46. Cotten CM, McDonald S, Stoll B, Goldberg RN, Poole K, Benjamin DK Jr. (2006) The association of third-generation cephalosporin use and invasive candidiasis in extremely low birth-weight infants. Pediatrics 118(2):717-722

47. Cotten CM, Taylor S, Stoll B, Goldberg RN, Hansen NI, Sanchez PJ et al (2009) Prolonged duration of initial empirical antibiotic treatment is associated with increased rates of necrotizing enterocolitis and death for extremely low birth weight infants. Pediatrics 123(1):58-66

48. Kuppala VS, Meinzen-Derr J, Morrow AL Schibler KR (2011) Prolonged initial empirical antibiotic treatment is associated with adverse outcomes in premature infants. J Pediatr 159(5):720-725

49. Van Houten MA, Uiterwaal CS, Heesen GJ, Arends JP, Kimpen JL (2001) Does the empiric use of vancomycin in pediatrics increase the risk for Gram-negative bacteremia? Pediatr Infect Dis J 20(2):171-177

50. de Man P, Verhoeven BA, Verbrugh HA, Vos MC, van den Anker JN (2000) An antibiotic policy to prevent emergence of resistant bacilli. Lancet 355(9208):973-978

51. Le J, Nguyen T, Okamoto M, McKamy S, Lieberman JM (2008) Impact of empiric antibiotic use on development of infections caused by extendedspectrum beta-lactamase bacteria in a neonatal intensive care unit. Pediatr Infect Dis J 27(4):314-318. https://doi.org/10.1097/INF.0b013e3181606850

52. Tripathi N, Cotten CM, Smith PB (2012) Antibiotic use and misuse in the neonatal intensive care unit. Clin Perinatol 39(1):61-68

53. Gilbert JA, Blaser MJ, Caporaso JG, Jansson JK, Lynch SV, Knight R(2018) Current understanding of the human microbiome. Nat Med 24(4):392-400. https://doi.org/10.1038/nm.4517

54. Cervantes J (2016) Use your antibiotics wisely. Consequences to the intestinal microbiome. FEMS Microbiol Lett. https://doi.org/10.1093/femsle/ fnw081

55. Vangay P, Ward T, Gerber JS, Knights D (2015) Antibiotics, pediatric dysbiosis, and disease. Cell
Host Microbe 17(5):553-564. https://doi.org/10. 1016/j.chom.2015.04.006

56. Hyun DY, Hersh AL, Namtu K, Palazzi DL, Maples HD, Newland JG et al (2013) Antimicrobial stewardship in pediatrics: how every pediatrician can be a steward. JAMA Pediatr 167(9):859-866. https:// doi.org/10.1001/jamapediatrics.2013.2241

57. Mukhopadhyay S, Puopolo KM (2017) Clinical and microbiologic characteristics of early-onset sepsis among very low birth weight infants: opportunities for antibiotic stewardship. Pediatr Infect Dis J 36(5):477-481. https://doi.org/10. 1097/inf.0000000000001473

58. Mukhopadhyay S, Sengupta S, Puopolo KM (2019) Challenges and opportunities for antibiotic stewardshipamong preterm infants. Arch Dis Child Fetal Neonatal Ed 104(3):F327-F332. https://doi. org/10.1136/archdischild-2018-315412

59. Cantey JB (2016) Optimizing the use of antibacterial agents in the neonatal period. Paediatr Drugs 18(2):109-122. https://doi.org/10.1007/s40272015-0161-1

60. Cantey JB, Huffman LW, Subramanian A, Marshall AS, Ballard AR, Lefevre Cetal (2017) Antibiotic exposure and risk for death or bronchopulmonary dysplasia in very low birth weight infants. J Pediatr 181:289-293e1. https://doi.org/10.1016/j.jpeds. 2016.11.002

61. Cantey JB, Sanchez PJ (2011) Prolonged antibiotic therapy for "culture-negative" sepsis in preterm infants: it's time to stop! J Pediatr 159(5):707-708. https://doi.org/10.1016/j.jpeds.2011.07.032

62. Cantey JB, Wozniak PS, Pruszynski JE, Sanchez PJ (2016) Reducing unnecessary antibiotic use in the neonatal intensive care unit (SCOUT): a prospective interrupted time-series study. Lancet Infect Dis 16(10):1178-1184. https://doi.org/10.1016/ s1473-3099(16)30205-5

63. Charani E, Castro-Sanchez E, Sevdalis N, Kyratsis Y, Drumright L, Shah N etal (2013) Understanding the determinants of antimicrobial prescribing within hospitals: the role of "prescribing etiquette". Clin Infect Dis 57(2):188-196. https://doi.org/10.1093/ cid/cit212

64. Klingenberg C, Kornelisse RF, Buonocore G, Maier RF, Stocker M (2018) Culture-negative earlyonset neonatal sepsis-at the crossroad between efficient sepsis care and antimicrobial stewardship. Front Pediatr 6:285. https://doi.org/10.3389/fped. 2018.00285

65. Steinmann KE, Lehnick D, Buettcher M, Schwendener-Scholl K, Daetwyler K, Fontana M et al (2018) Impact of empowering leadership on antimicrobial stewardship: a single center study in a neonatal and pediatric intensive care unit and a literature review. Front Pediatr 6:294. https://doi. org/10.3389/fped.2018.00294

66. Deutsche Gesellschaft für Neonatologie und Pädiatrische Intensivmedizin (GNPI), Deutsche Gesellschaft für Pädiatrische Infektiologie (DGPI), Deutsche Gesellschaft fürKinderund Jugendmedizin (DGKJ), Deutsche Gesellschaft für Gynäkologie udn geburtshilfe (DGGG). Bakterielle Infektion des Neugeborenen. AWMF Registernummer 024/008 (Entwicklungsstufe S2k). Arbeitsgemeinschaft Wissenschaftlicher Fachgesellschaften. 2018; Version 31. Jan. 2019.

67. Deutsche Gesellschaft für Pädiatrische Infektiologie (DGPI). S2k Leitlinie „Antibiotic Stewardship Konzeption und Umsetzung in der stationären Kinder-und Jugendmedizin" AWMF-Registernummer 048/15.2018;Version 1.Dez. 2018 